\title{
The impact of malocclusion and its treatment on the oral health related quality of life of adults, assessed using the Oral Health Impact Profile (OHIP-14)
}

\author{
Abstracted from \\ Andiappan M, Gao W, Bernabé E, Kandala NB, Donaldson AN. \\ Malocclusion, orthodontic treatment, and the Oral Health Impact Profile (OHIP-14): \\ Systematic review and meta-analysis. Angle Orthod 2015; 85: 493-500. \\ Address for correspondence: Manoharan Andiappan, Biostatistician and PhD student, \\ Biostatistics and Research Methods Centre, Floor 18, Guy's Tower, Dental Institute, \\ King's College London, London SE1 9RT, UK. E-mail: manoharan.1.andiappan@kcl.ac.uk; mano_757@yahoo.com
}

\section{Question: What is the impact of malocclusion and its treatment on OHIP-14 scores in adults?}

Data sources PubMed, Medline and Embase via OVID were searched up to November 2013. This was supplemented by a hand search of the reference lists of all relevant publications and recently published review articles. The search was restricted to the English language. Study selection Two review authors independently searched and selected the studies. Interventional or observational studies of individuals aged 15 years and above with either a malocclusion, or those who had undergone treatment for a malocclusion, were included. Studies must have included a control group without a malocclusion (for assessment of malocclusion impact), or a control group requiring treatment for correction of malocclusion (to assess the impact of treating malocclusion). Oral health related quality of life (OHRQoL) had to be measured using OHIP-14.

Data extraction and synthesis Two review authors independently carried out data abstraction and methodological quality assessment. Studies were divided into three groups based on the type of comparison groups; with and without malocclusion (independent design), the same group of individuals before and after treatment (pre-post design), and an orthodontically treated group with a group requiring treatment (treated-untreated groups design). For each group, data were pooled if the same type and details of OHIP-14 scores were reported. A Q test, $\mathrm{I}^{2}$ test and random-effects model were used. Begg and Egger tests and funnel plots were used to measure bias, including publication bias.

Results Twenty-five studies were included; eleven studies had an independent design, ten studies were classed as pre-post studies, and four studies were categorised as demonstrating a treated-untreated groups design. Data from four of the independently designed studies, which included a total of 892 patients, were pooled and a metaanalysis conducted. The data from three of the pre-post designed studies, with a total of 110 patients, were also pooled in a separate meta-analysis. The standardised mean difference (SMD) in OHIP-14 total score in the independently designed studies was $0.84(95 \%$ $\mathrm{Cl}: 0.25$ to 1.43$)$ and in the pre-post design group was 1.29 (95\% $\mathrm{Cl}: 0.67$ to 1.92 ). The studies in both meta-analyses demonstrated statistically significant levels of heterogeneity. There was also evidence of publication bias in the meta-analysis conducted using the independently designed studies.

Conclusions There are currently no high quality studies in the form of longitudinal or randomised controlled trials to assess the impact of malocclusion and its associated treatment on OHIP-14 scores respectively. Results from existing studies, which are predominantly cross-sectional, suggest that OHIP-14 scores are significantly lower in individuals without a malocclusion, indicating higher levels of OHRQoL. Similarly, longitudinal studies have shown that OHIP-14 scores are significantly lower after patients have received treatment for their malocclusion. The small number of trials, and their associated methodological concerns, limits the validity and reliability of the metaanalyses conducted, and the results obtained must be subjected to further scrutiny and confirmation.

\section{Commentary}

The assumed disadvantage posed by the presence of a malocclusion, and the benefits of correction using orthodontic treatment, have long been the subject of research interest. Research in this subject area has witnessed a paradigm shift, with patient reported outcome measures such as oral health related quality of life (OHRQoL) now forming the primary outcome measure in many trials.

The objective of this study was to systematically review the literature and synthesise evidence assessing the impact of malocclusion and its associated treatment on the OHRQOL of individuals aged 15 years and over, in studies using the OHIP14 questionnaire. Although this systematic review addressed an important subject, the aim was somewhat ambiguous. Whilst the desire of the authors to determine the impact of malocclusion on OHRQoL is warranted, they fail to explain whether they seek to determine the impact that undergoing treatment has on OHRQoL, or whether they aim to establish the influence that this has on OHRQoL once treatment is completed. There is an important distinction between these two questions, and without clearly highlighting the objectives of the systematic review, it is difficult to determine whether the inclusion and exclusion criteria for the study were appropriate. The systematic review also presented a number of methodological concerns. The authors presented a list of inclusion and exclusion criteria, explicitly stating that any studies lacking information regarding sample size, mean and standard deviation for the OHIP-14 total scores, or insufficient data to calculate these, would be excluded. Despite this however, of the twenty-five studies included in the review, they reported that only eight studies provided such data. It is therefore unclear why the authors included the remaining seventeen studies in the review. They also stated that studies must include a control group with individuals that do not possess a malocclusion, or a control 
group with individuals that require either orthodontic treatment or orthognathic surgery. However, they included ten studies that they classified as pre-post studies. These were longitudinal in nature and followed a single group of patients undergoing treatment over varying periods of time. These studies did not include longitudinal data from an untreated control group of patients with a similar condition, who might be, for example, on a waiting list for the same treatment. Such inconsistencies suggest that although comprehensive inclusion and exclusion criteria were presented for the systematic review, they were not strictly adhered to.

The authors reported that the methodological quality of studies was assessed using the STROBE checklists. However, they presented no data or information regarding this in their results. This makes interpretation of the methodological quality of the studies impossible. Where the impact of treating a malocclusion on OHIP14 scores was considered, the authors included both orthodontic treatment alone or in combination with orthognathic surgery. When classifying or analysing the studies, the authors made no attempt to differentiate between these two methods of treatment. It is widely accepted that orthognathic surgery is predominantly used to treat more severe forms of skeletal discrepancy, whilst orthodontic treatment alone is used to treat cases that demonstrate less severe skeletal discrepancies. The impact of each of these on OHIP-14 scores, and the changes observed on completion of treatment may vary considerably, and the authors made no attempt to consider or discuss this. The authors also failed to explicitly state the follow-up period in which OHIP-14 was assessed. A number of studies made assessments before and after orthognathic surgery was completed, but the authors of the review do not make it clear whether or not the post-surgical phase of orthodontic treatment was also completed. This raises the question about whether changes observed in OHIP-14 scores in these studies were reflective of the changes that occurred whilst undergoing treatment, or on its completion. Two of the three studies included in the pre-post study design meta-analysis assessed OHIP-14 scores before and after undergoing orthognathic surgery, whilst one study included scores once treatment was completed. With such variation in follow-up periods, doubts may be cast on the validity of the results from the meta-analysis.

The results of this systematic review and meta-analyses must be interpreted with caution due to the lack of high quality studies included. The four independent group studies comparing participants with and without malocclusion were all cross-sectional in nature. Current literature suggests that the presence of a malocclusion predominantly impacts on the emotional and social well-being domains of OHRQoL, ${ }^{1,2}$ and these, especially emotional well-being, are thought to be influenced by a multitude of factors including personality and self-esteem. ${ }^{3}$ As such factors can vary over time and be influenced by environmental factors, the impact of malocclusion on OHRQoL should ideally be assessed using longitudinal study designs that allow for the elimination of such confounding factors. The three studies with pre-post study designs were longitudinal in nature, but as they were assessing the impact of an intervention, a randomised controlled trial design may have been more appropriate. Moreover, the number of participants in these three studies was very small, with one study only recruiting 14 participants.

The present systematic review has made great effort in highlighting an important subject area and will no doubt serve as a driving force for continued research in the field. The authors sought to identify studies using OHIP-14 only, in order to allow the comparison of findings across the different studies, and, in order to allow for the quantitative synthesis of data, and their efforts in doing so should be commended. The methodological concerns raised may have perhaps been overcome had the study established more explicit aims from the outset. The lack of sufficient high quality evidence highlighted by the review should be noted and future studies should aim to address these.

\section{Practice point}

- The evidence presented is limited and no strongly supported conclusions can be drawn. However, based on current literature, it seems that malocclusion can have a negative impact on OHRQoL, and that its correction can lead to improved levels of OHRQoL.

- Although there appears to be a multitude of studies assessing OHIP-14 scores in patients with malocclusion and those who are or have undergone orthodontic treatment with or without orthognathic surgery, the reality is that there are so many variables in such studies, that no single study will be able to answer all the questions raised in this systematic review. Future systematic reviews should aim to address more specific questions, in order to produce meaningful results.

\section{Hanieh Javidi and Philip Benson Academic Unit of Oral Health and Development, University of Sheffield.}

1. Foster Page LA, Thomson WM, Jokovic A, Locker D. Validation of the Child Perceptions Questionnaire (CPQ 11-14). J Dent Res 2005; 84: 649-652.

2. O'Brien K, Wright JL, Conboy F, Macfarlane T, Mandall N. The child perception questionnaire is valid for malocclusions in the United Kingdom. Am J Orthod Dentofacial Orthop 2006; 129: 536-540.

3. Diener E, Oishi S, Lucas RE. Personality, culture, and subjective well-being: emotional and cognitive evaluations of life. Annu Rev Psychol 2003; 54: 403-425.

Evidence-Based Dentistry (2015) 16, 57-58. doi:10.1038/sj.ebd.6401100 